\title{
BMJ Open Precision orthotics: optimising ankle foot orthoses to improve gait in patients with neuromuscular diseases; protocol of the PROOF-AFO study, a prospective intervention study
}

\author{
Niels F J Waterval, ${ }^{1}$ Frans Nollet, ${ }^{1}$ Jaap Harlaar, ${ }^{2}$ Merel-Anne Brehm ${ }^{1}$
}

To cite: Waterval NFJ, Nollet F, Harlaar J, et al. Precision orthotics: optimising ankle foot orthoses to improve gait in patients with neuromuscular diseases; protocol of the PROOF-AFO study, a prospective intervention study. BMJ Open 2017;7: e013342. doi:10.1136/ bmjopen-2016-013342

- Prepublication history and additional material is available. To view please visit the journal (http://dx.doi.org/ 10.1136/bmjopen-2016013342).

Received 6 July 2016 Revised 10 November 2016 Accepted 4 January 2017

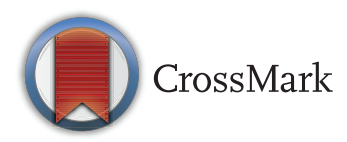

${ }^{1}$ Department of Rehabilitation, Academic Medical Center, University of Amsterdam, Amsterdam Movement Sciences, Amsterdam, The Netherlands

${ }^{2}$ Department of Rehabilitation Medicine, VU University Medical Center, Amsterdam Movement Sciences, The Netherlands

Correspondence to Niels F J Waterval; n.f.waterval@amc.uva.nl

\section{ABSTRACT}

Introduction: In patients with neuromuscular disorders and subsequent calf muscle weakness, metabolic walking energy cost (EC) is nearly always increased, which may restrict walking activity in daily life. To reduce walking EC, a spring-like ankle-footorthosis (AFO) can be prescribed. However, the reduction in EC that can be obtained from these AFOs is stiffness dependent, and it is unknown which AFO stiffness would optimally support calf muscle weakness. The PROOF-AFO study aims to determine the effectiveness of stiffness-optimised AFOs on reducing walking $\mathrm{EC}$, and improving gait biomechanics and walking speed in patients with calf muscle weakness, compared to standard, non-optimised AFOs. A second aim is to build a model to predict optimal AFO stiffness.

Methods and analysis: A prospective intervention study will be conducted. In total, 37 patients with calf muscle weakness who already use an AFO will be recruited. At study entry, participants will receive a new custom-made spring-like AFO of which the stiffness can be varied. For each patient, walking EC (primary outcome), gait biomechanics and walking speed (secondary outcomes) will be assessed for five stiffness configurations and the patient's own (standard) AFO. On the basis of walking $E C$ and gait biomechanics outcomes, the optimal AFO stiffness will be determined. After wearing this optimal AFO for 3 months, walking $\mathrm{EC}$, gait biomechanics and walking speed will be assessed again and compared to the standard AFO.

Ethics and dissemination: The Medical Ethics Committee of the Academic Medical Centre in Amsterdam has approved the study protocol. The study is registered at the Dutch trial register (NTR 5170). The PROOF-AFO study is the first to compare stiffness-optimised AFOs with usual care AFOs in patients with calf muscle weakness. The results will also provide insight into factors that influence optimal AFO stiffness in these patients. The results are necessary for improving orthotic treatment and will be disseminated through international peer-reviewed journals and scientific conferences.

\section{Strengths and limitations of this study}

- A wide variety of outcome measures is assessed to provide a broader view on the efficacy of stiffness optimised AFOs.

- The selection of the optimal AFO stiffness is based on objective walking energy cost and gait biomechanical measures.

- A limitation may be that only a limited range of stiffness is tested which may not include the optimal stiffness.

\section{INTRODUCTION}

Patients with neuromuscular disorders, such as poliomyelitis and Charcot-Marie-Tooth disease, frequently suffer from weakness or paresis of the calf muscles. Gait in calf muscle weakness is often characterised by excessive ankle dorsiflexion and persistent knee flexion during stance and by a reduced ankle push-off. ${ }^{1}$ These gait deviations nearly always lead to walking limitations such as instability, ${ }^{2}$ pain, ${ }^{3}{ }^{4}$ reduced speed ${ }^{56}$ and an increased walking energy cost (EC), ${ }^{5-7}$ which may restrict walking activity in daily life..$^{8-10}$

In normal gait, the calf muscles (gastrocnemius and soleus) prevent excessive ankle dorsiflexion, as the ground reaction force progresses over the foot in late stance. They create an eccentric force to restrain inclination of the shank, ${ }^{11}{ }^{12}$ preventing the ankle from collapsing in uncontrolled dorsiflexion. This is followed by a concentric contraction of the calf muscles during push-off, which assists in propelling the limb forward into swing and inducing knee flexion. ${ }^{11}{ }^{13}$ When the calf muscles are weak or paralysed, the forward progression of the shank will not be slowed down, which results in a rapid and uncontrolled ankle dorsiflexion, ${ }^{11}{ }^{14-16}$ moving the knee anteriorly and prolonging 
the time during which the ground reaction force passes behind the knee. This yields an increased external knee flexion moment and, hence, quadriceps overloading. ${ }^{11}$ Furthermore, as a consequence of calf muscle weakness, ankle push-off power is reduced, which may cause a shorter step length and single support time. ${ }^{13} 1417$ This reduces walking speed and, when compensated for, increases walking EC, ${ }^{59}$ which may lead to early fatigue during gait.

To improve gait and reduce walking EC, patients with calf muscle weakness can be provided with an orthosis that restrains ankle dorsiflexion, such as a carbon fibre dorsal leaf spring ankle-foot orthosis (DLS-AFO). ${ }^{18-21}$ When the ankle moves into dorsiflexion during late stance, this AFO acts like a spring and provides a plantar flexion moment at the ankle, thereby reducing the maximal dorsiflexion angle and shank inclination angle. $^{18} 22$ As a result of the reduced shank inclination, the knee is not constrained into flexion and the ground reaction force will progress more anterior in late stance. Consequently, the ground reaction force will not pass as far behind the knee as without the AFO, thereby reducing the external knee flexion moment during stance. ${ }^{1421}$ The spring-like properties of the DLS-AFO can also support ankle push-off by unleashing energy from the leaf in preswing that was loaded in the stance phase. ${ }^{17}{ }^{18}$ This energy takes over part of the ankle work during the gait cycle $^{17}$ and lowers soleus activity, ${ }^{23}$ thereby reducing the need for inefficient compensation strategies by patients with weak calf muscles. ${ }^{24}$ In healthy individuals, an exoskeleton based on this mechanism of storing and unleashing energy reduced the walking EC by $7 \% .^{25}$

The effectiveness of spring-like DLS-AFOs to reduce walking EC, however, is indicated to be stiffness dependent. $^{22}{ }^{25}$ Simulations in which AFO ankle stiffness was systematically varied demonstrated that with increasing stiffness walking EC first decreased, then increased; ${ }^{22}$ a trend also observed in healthy individuals wearing a spring-like exoskeleton. ${ }^{25}$ Moreover, in both studies, an optimal stiffness was found at which walking EC was minimal, supporting the idea that also in patients with calf muscle weakness there would be an optimal DLS-AFO stiffness that reduces walking EC the most.

In current clinical practice, a variety of off the shelf and custom-made AFOs and orthopaedic shoes for calf muscle weakness are provided, of which the effectiveness to reduce walking EC has not been secured. ${ }^{14}{ }^{26}$ Since the mechanical properties of these AFOs are generally fixed, it is not possible to individually adjust the orthotic stiffness. Hence, it may be assumed that common practice in providing AFOs for calf muscle weakness is biomechanically suboptimal in reducing walking EC and that stiffness-optimised DLS-AFOs will be more energy efficient in this respect, although this has not been studied yet. To reach consensus about the optimal AFO for people with calf muscle weakness, the effectiveness of stiffness-optimised AFOs compared to standard AFOs needs to be evaluated.
In addition, the factors that determine optimal DLS-AFO stiffness in calf muscle weakness need to be evaluated, assuming such stiffness exists. Patient characteristics such as degree of (calf) muscle weakness, ankle joint range of motion and body weight will most likely determine optimal AFO stiffness, ${ }^{14}{ }^{27}$ although this has not yet been investigated. If the factors that influence optimal stiffness are known, individual optimal stiffness may be computed based on pre-specified patient characteristics, which may contribute to improving AFO care in patients with neuromuscular disorders.

The study described in this design article will test the hypothesis that walking with a stiffness-optimised DLS-AFO is more energy effective compared to a standard, nonoptimised AFO for patients with neuromuscular disorders that demonstrate calf muscle weakness. Furthermore, our study aims to evaluate the effects of varying DLS-AFO stiffness on walking EC, gait biomechanics and speed and to create a simulation model to individually compute patient-dependent optimal DLS-AFO stiffness in calf muscle weakness.

\section{METHODS}

\section{Study design}

A prospective uncontrolled intervention study with three repeated measurements will be conducted to evaluate the effects of stiffness-optimised AFOs compared to standard, non-optimised AFOs. Measurements will be performed at baseline, walking with the currently used (standard) AFO (T1); directly after supplying the experimental AFO in five different stiffness (K) configurations $\left(\mathrm{T} 2_{\mathrm{K} 1}-\mathrm{T} 2_{\mathrm{K} 5}\right)$; and after a 3-month follow-up, walking with the selected stiffness-optimised experimental AFO ( $\mathrm{T} 3_{\text {Kopt }}$ ) (figure 1).

\section{Study population}

It is intended to include 37 patients with neuromuscular disorders with non-spastic paresis or weakness of the calf muscles, aged 18 and older and wearing an AFO. Although patients with calf muscle weakness often are able to walk without an AFO, they may need one to reduce instability, overuse symptoms and fatigue due to increased EC. Examples of neuromuscular disorders that can evoke calf muscle weakness and are eligible for this trial are poliomyelitis, Charcot-Marie-Tooth disease, inclusion body myositis, myotonic dystrophy and peripheral nerve injury. Patients will be recruited from the Dutch network of neuromuscular rehabilitation centres. The treating rehabilitation physician in these centres will select potentially eligible patients. Eligible patients will be invited to take part in the study by means of an information letter, including a response card. If the patient is willing to participate, inclusion and exclusion criteria (table 1) will be checked. When a patient meets the inclusion criteria, oral and written informed consent (consent form is attached as online supplementary file) will be obtained by a trained researcher. 


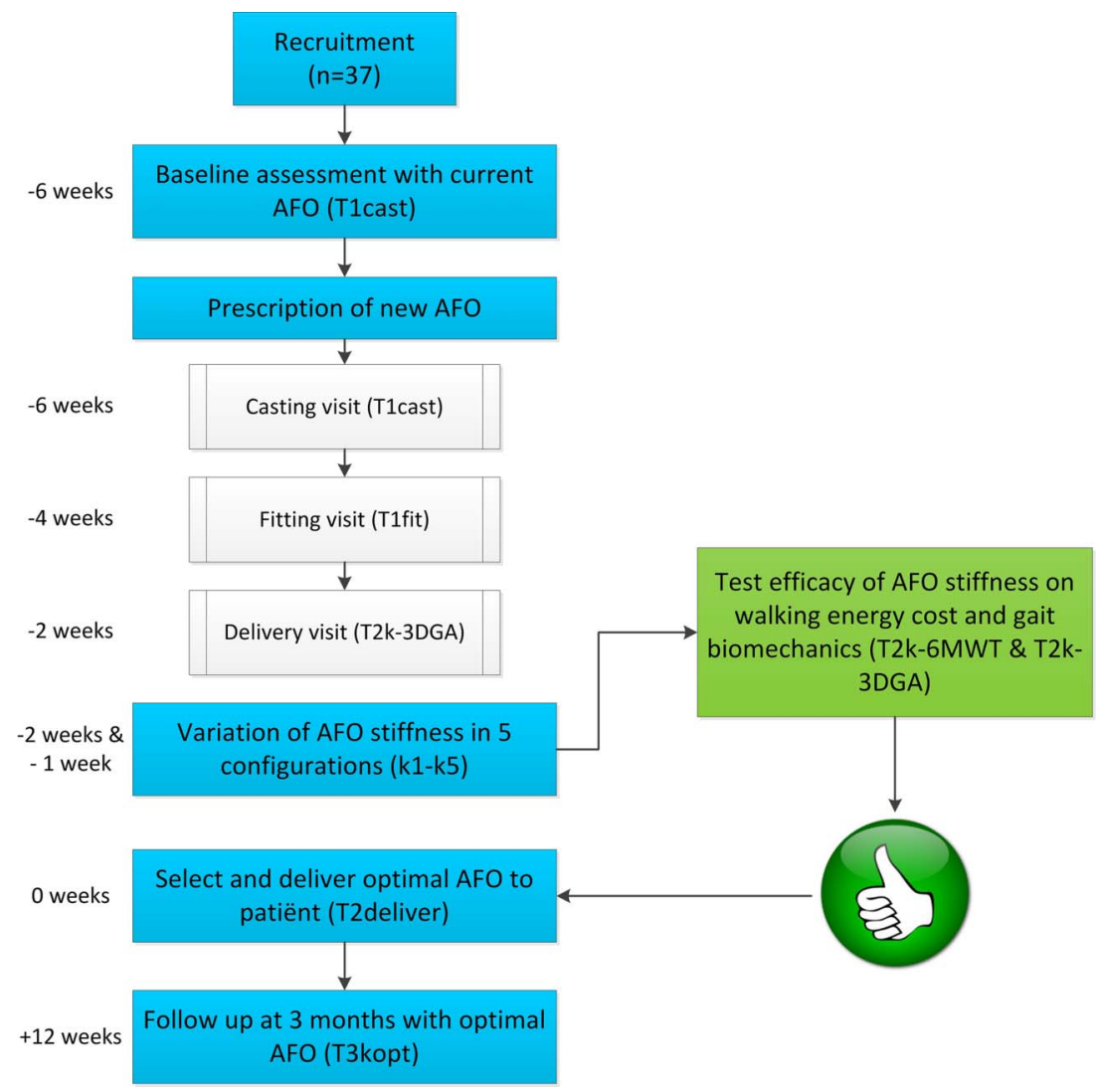

Figure 1 Schematic reproduction of the study design. After baseline measurements (T1), the subject's experimental AFO will be prescribed and fabricated (casting, fitting and delivery visit). Next, at the delivery visit, stiffness of the experimental AFO will be varied into five configurations $\left(\mathrm{T}_{\mathrm{K}_{1}}-\mathrm{T} 2_{\mathrm{K} 5}\right)$. Effects of each stiffness configuration will be evaluated, and subsequently, the subject's optimal AFO will be selected and supplied to the patient. Follow-up measurements for the selected optimal AFO $\left(\mathrm{T}_{\text {Kopt }}\right)$ will be performed 12 weeks later. AFO, ankle-foot-orthosis; $\mathrm{K}, \mathrm{AFO}$ stiffness; $\mathrm{K}_{1}$ (very flexible) through $\mathrm{K}_{5}$ (very stiff).

Table 1 Inclusion and exclusion criteria

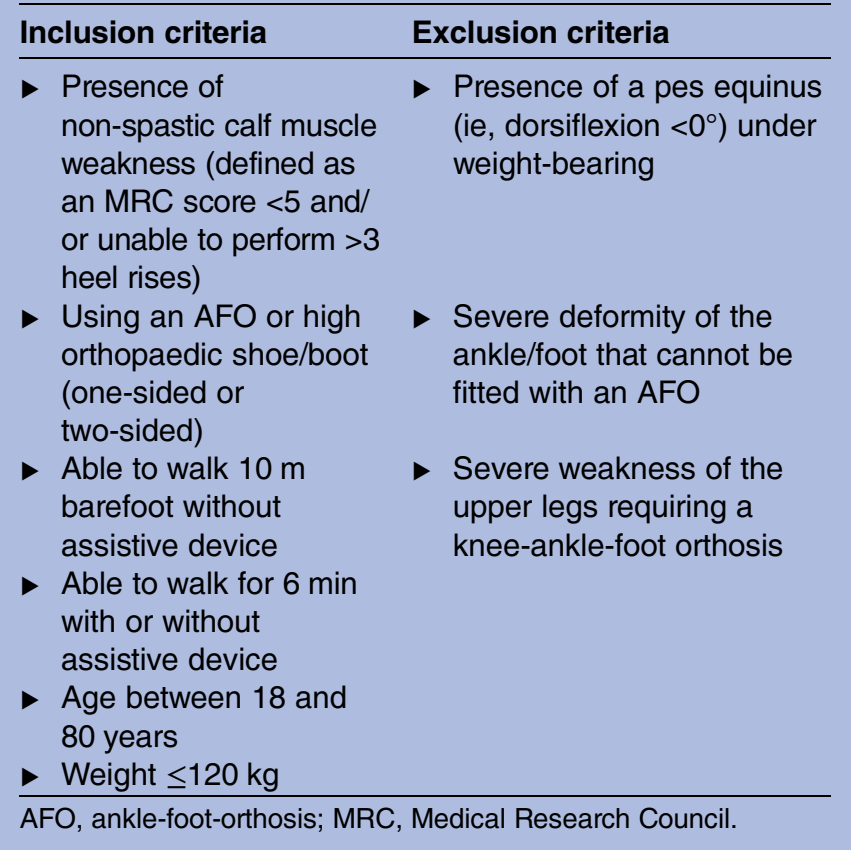

\section{Sample size}

The sample size for this study is based on a power analysis of the expected change in the primary outcome, metabolic walking EC $(\mathrm{J} / \mathrm{kg} / \mathrm{m})$. Walking EC in patients with neuromuscular disorders has been shown to be 40 $50 \%$ higher compared to healthy individuals. ${ }^{5} 72829$ According to the results of a previous study on the effect of AFOs in polio survivors, a reduction of $10 \%$ in walking EC $(0.52 \mathrm{~J} / \mathrm{kg} / \mathrm{m})$ is chosen as a clinical significant change. ${ }^{14}$ With an assumed SD of $0.70 \mathrm{~J} / \mathrm{kg} / \mathrm{m}$, a power of $90 \%$ and a significance level of 0.05 , a total of 34 patients are needed to detect a $10 \%$ change. Allowing for a dropout rate of $\sim 10 \%$, in total, 37 patients need to be included.

\section{Intervention}

\section{Standard AFO}

The standard AFO in our study may include any type of AFO or any type of high orthopaedic footwear with shaft reinforcement as prescribed in common practice for lower leg muscle weakness. 


\section{Experimental AFO}

The experimental AFO includes a newly fabricated DLS-AFO (made by OIM Noppe orthopedietechniek, Noordwijkerhout, The Netherlands), which will be worn in combination with the patients' own (orthopaedic) shoes. The DLS-AFO consists of a custom-made carbon foot part and calf casing, and a replaceable carbon fibre leaf spring (Carbon Ankle Seven, Ottobock, Duderstadt, Germany) (figure 2). As such, stiffness of the AFO can be varied within the same orthosis. For each patient, five springs will be evaluated (ranging in stiffness from very flexible (K1) to very stiff (K5)), which allows the selection of the stiffness with the maximal reduction in walking EC for a particular subject, referred to as the subject's optimal AFO. In case the experimental AFO harms the patient (eg, pain or other discomfort), the AFO will be adjusted until it fits. Furthermore, if needed, new orthopaedic footwear is provided. The intervention will only be terminated in case of urgent medical reasons or other urgent reasons.

\section{Compliance}

The optimal AFO will be worn by the patient according to an accommodation schedule that includes a gradual increase in the length of time the AFO is worn. Patients will be contacted 1 week after wearing the optimal AFO to check for adverse events (eg, pain or pressure sores).

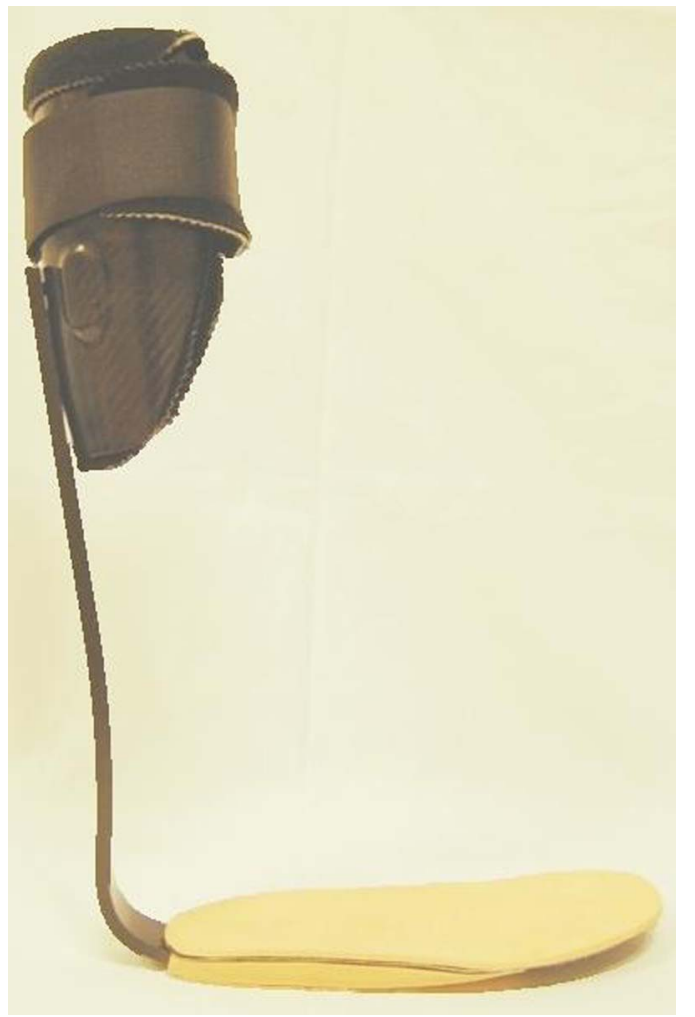

Figure 2 The experimental AFO. The stiffness of the AFO can be varied by exchanging the dorsal leaf spring. In total, five different springs (ranging in stiffness from very flexible to very stiff) will be assessed. AFO, ankle-foot-orthosis.
If the patient has no symptoms, the follow-up period will start. To measure the patients' compliance with wearing the AFO during the follow-up period, an adherence to treatment monitor (ODM, Academic Medical Center, Amsterdam, the Netherlands) will be fitted inside the calf casing of the AFO. The adherence monitor is a small temperature-based monitoring system, consisting of two temperature sensors, which allows us to determine when the AFO is worn based on the temperature difference between the sensors. ${ }^{30}$ Compliance with wearing the optimal AFO will be assessed for seven consecutive days during the last week of the follow-up period. Patients are discouraged from wearing their standard AFO during follow-up. During the baseline period, compliance with the standard AFO will be assessed.

\section{Study outcomes}

Study outcomes will be assessed at baseline (T1), directly after supplying the experimental AFO (T2), and after a 3-month follow-up (T3).

\section{Primary outcome}

The primary outcome of this study is walking EC, defined as the metabolic energy used per distance covered. Walking EC will be determined during a 6-min walk test (6MWT) at a self-selected comfortable speed on a 35-m indoor oval track. During the test, breath-by-breath oxygen uptake $\left(\mathrm{VO}_{2}\right)$ and carbon dioxide production $\left(\mathrm{VCO}_{2}\right)$ values will be assessed with the Cosmed $\mathrm{K}^{2} \mathrm{~B}^{2}$ portable gas analyser (Cosmed, Rome, Italy). Mean steady state $\mathrm{VO}_{2}, \mathrm{VCO}_{2}$ and walking speed values will be determined between the fourth and sixth minutes of the walk test with a custom-written Matlab script (V.2015; MathWorks, Natick, Massachusetts, USA). On the basis of these values, the walking EC per metre will be calculated, according to the following formula: $\left(\left(\left(4.940 \times\left(\mathrm{VCO}_{2}\right)\right.\right.\right.$ $\left.\left.\left.\mathrm{VO}_{2}\right)+16.040\right) \times \mathrm{VO}_{2}\right) /$ walking speed in $\mathrm{m} / \mathrm{s}$ ) where $\mathrm{VCO}_{2}$ and $\mathrm{VO}_{2}$ are in $\mathrm{ml} / \mathrm{kg} / \mathrm{min} .{ }^{31}$ Previously, it has been shown that walking EC can be reliably assessed in patients with walking difficulties. ${ }^{732} 33$

\section{Secondary outcomes}

Secondary outcomes include gait biomechanics, daily step activity, walking speed (assessed during the 6-min walk test), perceived physical functioning (assessed with the 36-Item Short-Form Health Survey (SF-36) ${ }^{34}$ ) interference of fatigue with functioning (assessed with the Fatigue Severity Scale $(\mathrm{FSS})^{35}$ ) and AFO satisfaction (assessed using a 10-point numeric rating scale). Two of these measures (gait biomechanics and daily step activity) are further explained below.

Gait biomechanics will be measured during a 3D gait analysis with a $100 \mathrm{~Hz}$ eight-camera 3D motion capture system (VICON MX 1.3). Reflective markers will be placed on the body according to the Plug-in Gait model together with four additional markers to measure bending of the dorsal leaf and movement of the AFO relative to the shank. After a static calibration, patients 
will be asked to walk over a 12-m long walkway in the gait laboratory. Simultaneously, ground reaction forces from two adjacent force plates within the walkway under the left and right feet will be recorded at $1000 \mathrm{~Hz}$ (OR6-7; AMTI, Watertown, Massachusetts, USA). For each walking condition, three valid gait trials will be collected. A trial is considered valid if the patient stands on a force plate with one foot, and all markers are visible from heel strike until ipsilateral heel strike, thereby collecting a full gait cycle for both legs. For each condition, joint angles, net joint moments and joint powers around the hip, knee and ankle are calculated and time normalised to the gait cycle $(0-100 \%)$. Finally, the three trials are averaged and specific outcome parameters such as peak dorsiflexion angle, peak ankle power and peak knee extension angle and moment at midstance will be calculated. These outcomes will be compared between different AFO configurations (eg, T2k1 and T2k2) and measurement moments (eg, T1 vs T2 and T3).

Daily step activity will be measured for seven consecutive days with the StepWatch3 Activity Monitor 3.0 (Stepwatch), which is a pedometer that is worn around the ankle. The Stepwatch records the number of steps per minute over a broad range of step cadences and has been used in patients with a neuromuscular disorder before. ${ }^{36}{ }^{37}$ Patients will be instructed not to remove the Stepwatch during the 7 days of measurement. For appropriate data cleaning and data interpretation, participants will be asked to note their activity programme during the day in a diary (eg, time of getting up and type of activities during the day). With the data of the Stepwatch, activity diary and adherence monitor, daily step activity while walking with and without AFO and daily step activity while walking inside and outside the house will be calculated.

\section{Additional outcomes}

\section{Patient characteristics}

Demographics (eg, sex, ethnicity) and anthropometrics (body weight and height) of the patients will be recorded. Furthermore, manual muscle strength of the ankle plantar flexors and dorsal flexors, knee flexors and extensors and hip flexors, extensors, abductors and adductors will be assessed and scored according to the Medical Research Council (MRC) Scale. ${ }^{38}$ In addition, quantitative strength scores of the ankle plantar flexors, ankle dorsal flexors, knee flexors and knee extensors will be assessed with a fixed dynamometer (System 3 PRO; BIODEX, Shirley, New York, USA). To quantify the intramuscular fat fraction and skeletal muscle architecture, patients will undergo a diffusion tensor imaging (DTI) scan of the lower legs.

\section{AFO stiffness}

Stiffness of the AFO-footwear combination around the forefoot and the ankle will be measured with the Bi-articular Reciprocal Universal Compliance Estimator (BRUCE), which is an instrument to measure AFO mechanical properties. ${ }^{39}$ Information on the AFOs' mechanical properties is needed to develop the AFO treatment algorithm and simulation model for optimal AFO stiffness. ${ }^{40}$

\section{Study procedures}

Patients will visit the hospital six times within a period of 16-20 weeks. An overview of the visits and measurements per visit is given in figure 1 and table 2, respectively.

During the first visit (casting visit $\left(\mathrm{T} 1_{\text {cast }}\right)$ ), inclusion and exclusion criteria will be checked. After a baseline assessment of demographics, anthropometrics and muscle strength, patients will be casted for their experimental AFO. Between the first and second visits, daily step activity will be measured with the StepWatch, the adherence monitor and the activity diary.

During the second visit, $\mathrm{T}_{\text {fit }}$, walking $\mathrm{EC}$ and speed will be assessed for walking with shoes only and the patients' standard AFO. Furthermore, patients will be asked to fill in the SF36 and FSS questionnaires, and a DTI scan of the lower legs will be conducted. The scan will be made before or at least $30 \mathrm{~min}$ after the walking test to avoid interference of additional blood flow and muscle damage with the DTI scan.

At the third visit, the experimental AFO will be delivered. Fitting and alignment of the AFO will be checked and, if necessary, corrected by the orthotist. Patients can walk up and down a hallway to adjust to the new AFO. After patients feel comfortable with the new AFO, they will be tested for gait biomechanics while walking barefoot, with shoes only, their current $\mathrm{AFO}$ and the experimental $\mathrm{AFO}$ in five stiffness configurations $\left(\mathrm{T} 2_{\mathrm{k}-3 \mathrm{DGA}}\right)$. The order of stiffness configurations will be randomly assigned, using a balanced block randomisation for all possible sequences, to ensure that the same number of patients is allocated to each sequence. The randomisation is performed per Matlab script (V.2015, MathWorks). Between the different conditions, patients will be allowed enough rest and have a $5 \mathrm{~min}$ acclimation period in which they can walk with and adapt to the new stiffness.

One week after the assessment of gait biomechanics, walking EC and speed will be measured for the five stiffness configurations of the experimental AFO $\left(\mathrm{T} 2_{\mathrm{K}-6 \mathrm{MWT}}\right)$. An evaluation of all AFO stiffness configurations will allow the selection of the stiffness with the maximal benefit for a particular subject (explained below), referred to as the subject's optimal AFO, which will be provided to the patient at the fifth visit $\left(\mathrm{T} 2_{\text {deliver }}\right)$. During this fifth visit, the ankle and forefoot stiffness of the experimental AFO (all five configurations) and the patients' standard AFO will be measured with the BRUCE device.

One week after providing the optimal AFO, patients will be contacted to check for adverse events. If the patient has no symptoms, the follow-up period will start, which will last until the next study visit, 12 weeks later. If patients report any adverse event during the follow-up period, the adverse event will be recorded and checked on regularly. At the start of the follow-up visit $\left(\mathrm{T} 3_{\mathrm{Kopt}}\right)$, patients are asked about adverse events within the 
Table 2 Overview of measurements per visit

\begin{tabular}{|c|c|c|c|c|c|c|c|}
\hline & & $\begin{array}{l}\mathrm{T}_{\text {cast }} \\
\text { Visit } 1\end{array}$ & $\begin{array}{l}\mathrm{T} 1_{\text {fit }} \\
\text { Visit } 2\end{array}$ & $\begin{array}{l}\text { T2 } \text { k-3DGA }^{*} \\
\text { Visit } 3\end{array}$ & $\begin{array}{l}\text { T2 }_{\text {k-6MWT }}{ }^{*} \\
\text { Visit } 4\end{array}$ & $\begin{array}{l}\text { T2 deliver } \\
\text { Visit } 5\end{array}$ & $\begin{array}{l}\text { T3 } \text { k-opt } \\
\text { Visit } 6\end{array}$ \\
\hline \multicolumn{8}{|l|}{ Primary outcome } \\
\hline \multicolumn{8}{|l|}{ Secondary outcomes } \\
\hline Gait biomechanics & 3DGA† & & & $x$ & & & $x$ \\
\hline Walking speed & 6MWT & & $x$ & & $x$ & & $x$ \\
\hline Daily step activity & SAM $\ddagger$ & & $x$ & & & & $x$ \\
\hline Adherence & ODM & & $x$ & & & & $x$ \\
\hline \multirow[t]{2}{*}{ Satisfaction } & NRS & $x$ & & $x$ & & & $x$ \\
\hline & LiS & & & & & & $x$ \\
\hline \multicolumn{8}{|l|}{ Additional outcomes } \\
\hline Demographics & Intake & $x$ & & & & & \\
\hline AFO stiffness & BRUCE & & & & & $x$ & \\
\hline \multicolumn{8}{|c|}{ 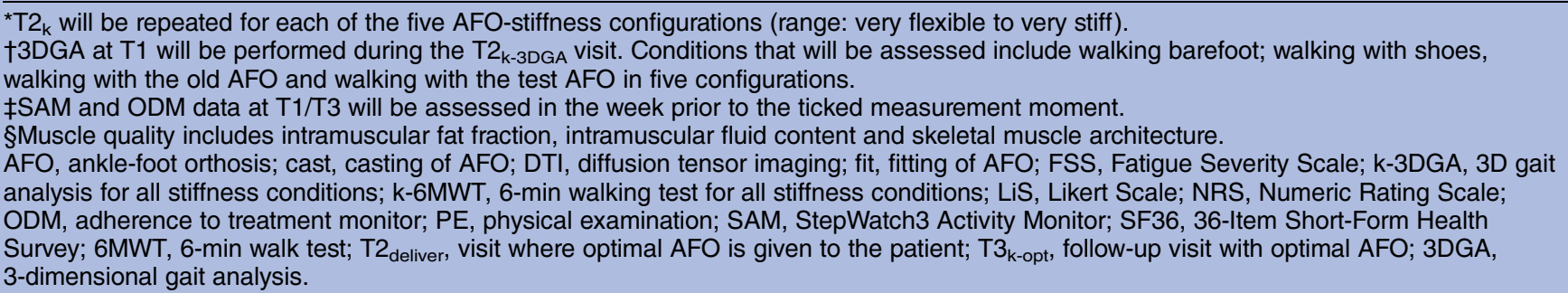 } \\
\hline
\end{tabular}

follow-up period that were not previously reported. During this visit, walking EC, walking speed, gait biomechanics, perceived physical functioning, perceived fatigue and satisfaction with the optimal AFO will be assessed. Furthermore, compliance and daily step activity will be assessed for the optimal AFO in the week prior to the follow-up measurement.

\section{Selection of optimal AFO}

After the $\mathrm{T} 2_{\mathrm{K}-6 \mathrm{MWT}}$ visit, the optimal AFO stiffness will be selected based primarily on walking EC in view of walking speed and secondarily on the gait pattern (see figure 3). The procedure starts by sorting the measured stiffness configurations by walking EG outcome. All conditions that have $\mathrm{a} \geq 5 \%$ higher EC compared to the condition with the lowest recorded EC will be excluded from the selection procedure, unless the walking speed is $\geq 5 \%$ higher compared to the speed of the condition with the lowest EC. The $5 \%$ range for the EC is chosen because of the mediocre precision of this measure. ${ }^{7}$ The reason that walking speed is taken into account is because this is an important parameter for daily activities. ${ }^{41} 42$ Subsequently, three assessors will independently evaluate the gait pattern of the remaining configurations and pick the configuration that normalises the gait pattern the most according to three predefined parameters: (1) peak dorsiflexion angle in late stance, (2) peak knee extension angle during single support and (3) peak ankle power. Disagreements in assignment of the optimal AFO will be resolved with a consensus procedure.

In case a patient wears AFOs bilaterally, both AFOs will be optimised. If the difference in MRC score for the calf muscles is $<1$ grade, EC and gait biomechanics will be assessed with the same AFO stiffness on both legs because no differences in optimal stiffness between the legs are expected. Optimisation will be performed for both legs simultaneously using the aforementioned procedure (see figure 3), and patients are always provided with the same AFO stiffness for both legs. In case the MRC score of the calf muscles differs more than one grade between legs, both AFOs will be optimised separately. First, the AFO for strongest leg will be optimised solely based on a gait analysis where the experimental AFO is worn on the strongest leg and the patient's own AFO on the weakest leg. After the AFO for the strongest leg has been optimised, EC and gait biomechanics will be assessed using the optimal AFO on the strongest leg and altering AFO stiffness on the weaker leg. On the basis of these data, the AFO for the weakest leg will be optimised using the procedure described above (see figure 3).

\section{Statistical analyses}

Data for all patients will be coded and entered into a secured database, OpenClinica. In OpenClinica, data will be checked using validation rules and cleaned when 


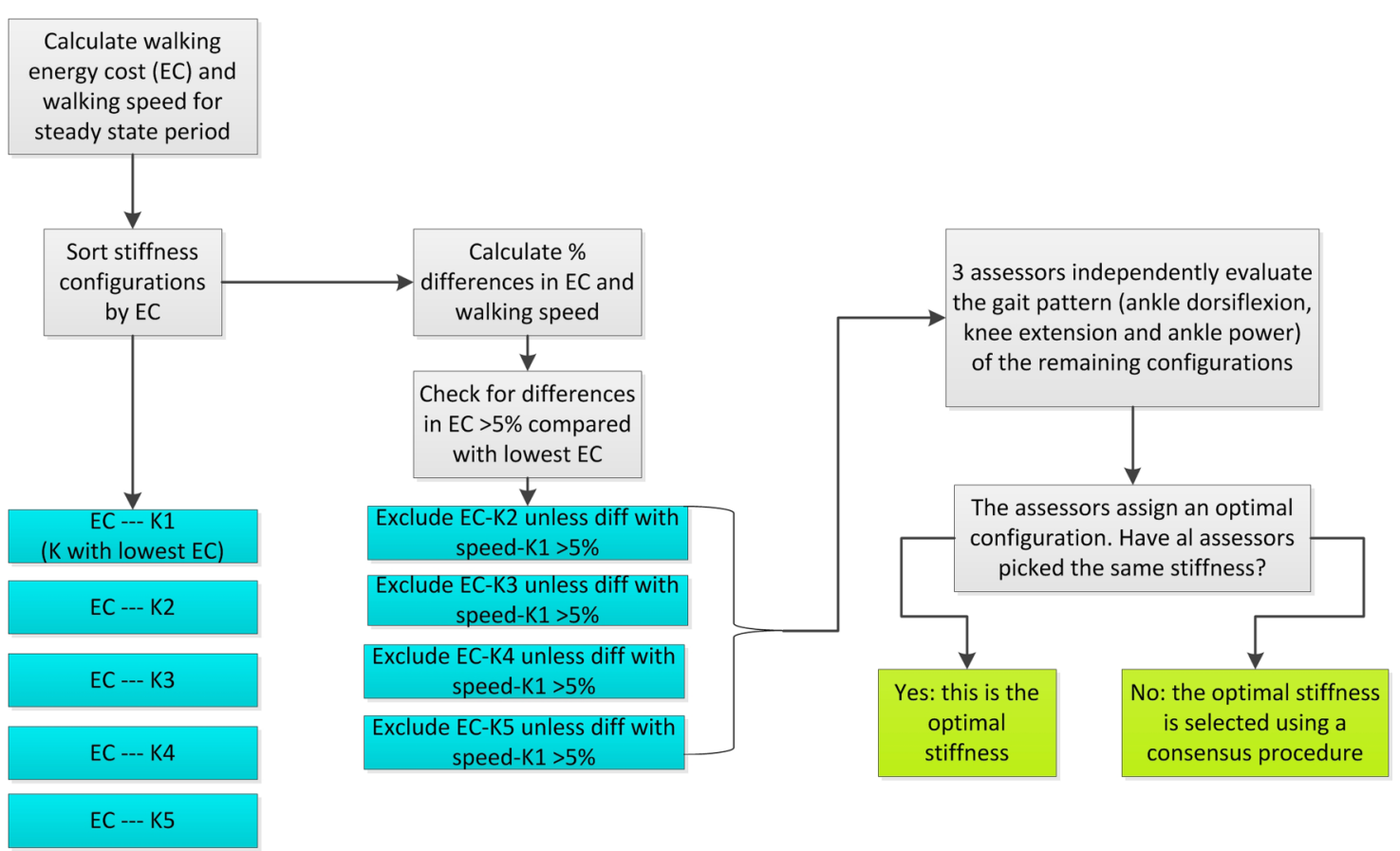

Figure 3 Selection procedure of the optimal AFO stiffness. The selection of the optimal AFO starts by sorting the measured stiffness configurations by walking energy cost outcome. All conditions that have a $5 \%$ higher EC compared to the lowest recorded EC will be excluded from the selection procedure, unless the walking speed is $5 \%$ higher compared to the speed of the condition with the lowest EC. In the second step, three assessors will independently evaluate the gait pattern of the remaining configurations and pick the configuration that normalises the gait pattern the most according to three predefined gait parameters. EC, energy cost.

data are incorrect before statistical analysis. If patients are lost to follow-up or terminated the study, recorded data will be used for the analysis. Demographic variables and disease characteristics of participants will be summarised using descriptive statistics. In addition, means, SDs and 95\% CIs for all outcome measures will be presented.

Evaluation of treatment efficacy of the subject's optimal AFO will be based on analyses of preintervention/post-intervention differences in the primary and secondary outcomes. Means of baseline measurements (T1) will be compared to the postintervention measurements ( $2_{6}{ }_{6 \mathrm{MWT}}$ and $\left.\mathrm{T} 2_{\mathrm{K}-3 \mathrm{DGA}}\right)$ and follow-up measurements $\left(\mathrm{T} 3_{\text {Kopt }}\right)$ using a Linear Mixed Model for repeated measures.

Computation of patient-dependent optimal DLS-AFO stiffness will be performed with simulation modelling. Development of the simulation models will be a conceptual follow-up on the work of Bregman et al. ${ }^{22}$ Baseline data on body weight, muscle strength, skeletal muscle architecture, intramuscular fat fraction, gait biomechanics and AFO stiffness will be used to parameterise (individualise) the model. Data on gait biomechanics at follow-up will be used for validation of the model.

\section{DISCUSSION}

The PROOF-AFO study will evaluate the effectiveness of stiffness-optimised DLS-AFOs on reducing walking EC and improving gait biomechanics and walking speed in patients with calf muscle weakness compared to standard AFOs. Furthermore, it aims to create a computational model to determine the optimal AFO stiffness for each patient, assuming such stiffness exists. This study captures several important strengths.

First, our study uses a stiffness-adjustable AFO design by a replaceable carbon fibre leaf spring, which enables the stiffness of the AFO to be varied within the same custom-made orthosis. This is an important advantage, as it allows a comparison of the efficacy between different AFO stiffness configurations, while minimising confounding factors, such as differences in alignment and footplate length or stiffness. Furthermore, the AFO is fabricated with standardised sizes of components that can be easily implemented in daily practice. This ensures direct improvement of AFO care if stiffness-optimised AFOs are more effective compared to standard AFOs currently used in clinical practice. Although we measure five different stiffnesses over a broad range, the optimal stiffness may not be included, which is a limitation of our study. We use multiple outcome measures to compare the usual care AFO with the optimised experimental AFO on different levels of the International Classification of Functioning, Disability and Health, providing a unique data set. With this data set, a broader view on the efficacy of stiffness-optimised AFOs on gait biomechanics and the impact of these AFOs on patients' daily life can be assessed. ${ }^{40}$ In addition, the large data set will provide 
input for creating and adjusting a musculoskeletal model in such a way that optimal AFO stiffness may be computed. This would enable clinicians to provide each patient with an optimal AFO stiffness, based on their individual characteristics.

In conclusion, the PROOF-AFO study will be the first to compare the effectiveness of stiffness-optimised AFOs with standard AFOs in patients with neuromuscular disorders exhibiting calf muscle weakness. The ECs of walking will be the primary outcome of this study, but the evaluation includes multiple outcome measures, which allows us to give an extensive comparison between AFOs with different stiffnesses and to create a simulation model to compute optimal stiffness. These results may provide new insights about how AFO stiffness influences gait in patients with calf muscle weakness, but they may also directly improve AFO care by providing a computational model for individually determining optimal stiffness that can be applied in clinical practice.

\section{DISSEMINATION}

The Medical Ethics Committee of the Academic Medical Center (AMC) has approved the study protocol, and the study will be performed at the Department of Rehabilitation of the AMC in Amsterdam, The Netherlands. The trial is registered at the Dutch Trial Register (NTR 5170) and will be carried out according to good clinical practice guidelines. Patients receive a study number, which will be used on all forms instead of names. Forms will be stored in a locked cabinet to assure anonymity. Only persons involved in the study have access to these forms before and after the study. A steering committee oversees the progress of the study, while monitoring will be performed by an independent monitor of the AMC. Aspects that will be monitored will include: inclusion rate; trial master file; informed consent process; inclusion and exclusion criteria; source data verification; safety reporting; investigational product; trial procedures and closing and reporting. Important protocol changes will be recorded (a new protocol version number will be assigned) and reported to the Medical Ethics Committee. The study is insured in case patients are harmed by participation in the study. After completion of the study, a manuscript with positive as well as negative or inconclusive results will be submitted to a peer-reviewed journal and presented at scientific conferences. Furthermore, the study data sets and statistical codes will be available on request. Participants will be informed about the results by a newsletter.

Contributors M-AB, FN and $\mathrm{JH}$ conceived this study. M-AB, FN, JH and NFJW contributed to the conception of the study design and participated in logistical planning of the study. NFJW is responsible for data acquisition and drafted the manuscript. M-AB, FN and $\mathrm{JH}$ critically appraised the draft versions of this manuscript and approved the final version.

Funding This study was supported by the Prinses Beatrix Spierfonds, grant number [W.OR 14-21]. OIM Noppe Orthopedie is thanked for manufacturing the intervention AFOs and Otto Bock for providing the Carbon Ankle Seven leaf springs.
Competing interests None declared.

Provenance and peer review Not commissioned; externally peer reviewed.

Data sharing statement Data sets and statistical codes will be available on request.

Open Access This is an Open Access article distributed in accordance with the Creative Commons Attribution Non Commercial (CC BY-NC 4.0) license, which permits others to distribute, remix, adapt, build upon this work noncommercially, and license their derivative works on different terms, provided the original work is properly cited and the use is non-commercial. See: http:// creativecommons.org/licenses/by-nc/4.0/

\section{REFERENCES}

1. Perry J, Burnfiel JM. Gait analysis; normal and pathological function. 2nd edn. Thorofare: SLACK Incorporated, 2010.

2. Bickerstaffe A, Beelen A, Nollet F. Circumstances and consequences of falls in polio survivors. $J$ Rehabil Med 2010;42:908-15.

3. Nollet $F$, Beelen A, Prins $M H$, et al. Disability and functional assessment in former polio patients with and without postpolio syndrome. Arch Phys Med Rehabil 1999;80:136-43.

4. Abresch RT, Carter GT, Jensen MP, et al. Assessment of pain and health-related quality of life in slowly progressive neuromuscular disease. Am J Hosp Palliat Care 2002;19:39-48.

5. Menotti F, Felici F, Damiani A, et al. Charcot-Marie-Tooth $1 \mathrm{~A}$ patients with low level of impairment have a higher energy cost of walking than healthy individuals. Neuromuscul Disord 2011;21:52-7

6. Brehm MA, Beelen A, Doorenbosch CA, et al. Effect of carbon-composite knee-ankle-foot orthoses on walking efficiency and gait in former polio patients. J Rehabil Med 2007;39:651-7.

7. Brehm MA, Nollet F, Harlaar J. Energy demands of walking in persons with postpoliomyelitis syndrome: relationship with muscle strength and reproducibility. Arch Phys Med Rehabil 2006;87:136-40.

8. Horemans $\mathrm{HJ}$, Bussmann JB, Beelen A, et al. Walking in postpoliomyelitis syndrome: the relationships between time-scored tests, walking in daily life and perceived mobility problems. $J$ Rehabil Med 2005;37:142-6.

9. Menotti F, Laudani L, Damiani A, et al. Amount and intensity of daily living activities in Charcot-Marie-Tooth 1A patients. Brain Behav 2014;4:14-20.

10. McCrory MA, Kim HR, Wright NC, et al. Energy expenditure, physical activity, and body composition of ambulatory adults with hereditary neuromuscular disease. Am J Clin Nutr 1998;67:1162-9.

11. Perry J, Clark D. Biomechanical abnormalities of post-polio patients and the implications for orthotic management. Neurorehabilitation 1997;8:119-38.

12. Kepple TM, Siegel KL, Stanhope SJ. Relative contributions of the lower extremity joint moments to forward progression and support during gait. Gait Posture 1997;6:1-8

13. Lehmann JF. Push-off and propulsion of the body in normal and abnormal gait. correction by ankle-foot orthoses. Clin Orthop Relat Res 1993;288:97-108.

14. Ploeger HE, Bus SA, Brehm MA, et al. Ankle-foot orthoses that restrict dorsiflexion improve walking in polio survivors with calf muscle weakness. Gait Posture 2014;40:391-8.

15. Rijken NH, van Engelen BG, de Rooy JW, et al. Gait propulsion in patients with facioscapulohumeral muscular dystrophy and ankle plantarflexor weakness. Gait Posture 2015;41:476-81.

16. Lehmann JF, Condon SM, de Lateur BJ, et al. Gait abnormalities in tibial nerve paralysis: a biomechanical study. Arch Phys Med Rehabil 1985;66:80-5.

17. Bregman DJ, Harlaar J, Meskers CG, et al. Spring-like Ankle Foot Orthoses reduce the energy cost of walking by taking over ankle work. Gait Posture 2012;35:148-53.

18. Desloovere K, Molenaers G, Van Gestel L, et al. How can push-off be preserved during use of an ankle foot orthosis in children with hemiplegia? A prospective controlled study. Gait Posture 2006;24:142-51.

19. Wolf $\mathrm{SI}$, Alimusaj M, Rettig O, et al. Dynamic assist by carbon fiber spring AFOs for patients with myelomeningocele. Gait Posture 2008;28:175-7.

20. Phillips MF, Robertson Z, Killen B, et al. A pilot study of a crossover trial with randomized use of ankle-foot orthoses for people with Charcot-Marie-Tooth disease. Clin Rehabil 2012;26:534-44.

21. Bartonek Â, Eriksson M, Gutierrez-Farewik EM. Effects of carbon fibre spring orthoses on gait in ambulatory children with motor disorders and plantarflexor weakness. Dev MedChild Neurol 2007;49:615-20. 
22. Bregman D, Van der Krogt M, De Groot V, et al. The effect of ankle foot orthosis stiffness on the energy cost of walking: a simulation study. Clin Biomech 2011;26:955-61.

23. Arch ES, Stanhope SJ, Higginson JS. Passive-dynamic ankle-foot orthosis replicates soleus but not gastrocnemius muscle function during stance in gait: insights for orthosis prescription. Prosthet Orthot Int 2015:40:606-16.

24. Collins SH, Kuo AD. Recycling energy to restore impaired ankle function during human walking. PLoS One 2010;5:e9307.

25. Collins $\mathrm{SH}$, Wiggin MB, Sawicki GS. Reducing the energy cost of human walking using an unpowered exoskeleton. Nature 2015;522:212-15.

26. Menotti F, Laudani L, Damiani A, et al. An anterior ankle-foot orthosis improves walking economy in Charcot-Marie-Tooth type $1 \mathrm{~A}$ patients. Prosthet Orthot Int 2014;38:387-92.

27. Guillebastre B, Calmels P, Rougier PR. Assessment of appropriate ankle-foot orthoses models for patients with charcot-marie-tooth disease. Am J Phys Med Rehabil 2011;90:619-27.

28. Waters R, Lunsford B. Energy expenditure of normal and pathological gait: application to orthotic prescription. In: Goldberg B, Hsu JD, eds. Atlas of orthotics, 2nd edn. St Louis (MO): Mosby. 1985:151-9.

29. Waters R, Yakura J, Adkins R, et al. Determinants of gait performance following spinal cord injury. Arch Phys Med Rehabil 1989; 70:811-8.

30. Bus SA, Waaijman R, Nollet F. New monitoring technology to objectively assess adherence to prescribed footwear and assistive devices during ambulatory activity. Arch Phys Med Rehabil 2012;93:2075-9.

31. Garby L, Astrup A. The relationship between the respiratory quotient and the energy equivalent of oxygen during simultaneous glucose and lipid oxidation and lipogenesis. Acta Physiol Scand 1987;129:443-4.

32. Danielsson A, Willén C, Sunnerhagen KS. Measurement of energy cost by the physiological cost index in walking after stroke. Arch Phys Med Rehabil 2007;88:1298-303.
33. da Cunha-Filho IT, Henson H, Wankadia S, et al. Reliability of measures of gait performance and oxygen consumption with stroke survivors. J Rehabil Res Dev 2003;40:19.

34. McHorney CA, War JEJr, Lu JR, et al. The MOS 36-item Short-Form Health Survey (SF-36): III. Tests of data quality, scaling assumptions, and reliability across diverse patient groups. Med Care 1994:40-66.

35. Krupp LB, LaRocca NG, Muir-Nash J, et al. The fatigue severity scale. Application to patients with multiple sclerosis and systemic lupus erythematosus. Arch Neurol 1989;46:1121-3.

36. Klein MG, Braitman LE, Costello R, et al. Actual and perceived activity levels in polio survivors and older controls: a longitudinal study. Arch Phys Med Rehabil 2008;89:297-303.

37. Busse ME, van Deursen RW, Wiles CM. Real-life step and activity measurement: reliability and validity. J Med Eng Technol 2009;33:33-41.

38. Council MR. Aids to examination of the peripheral nervous system. Memorandum no. 45. London: Her Majesty's Stationary Office, 1976.

39. Bregman DJ, Rozumalski A, Koops D, et al. A new method for evaluating ankle foot orthosis characteristics: BRUCE. Gait Posture 2009;30:144-9.

40. Harlaar J, Brehm M, Becher JG, et al. Studies examining the efficacy of ankle foot orthoses should report activity level and mechanical evidence. Prosthet Orthot Int 2010;34:327-35.

41. Vermeulen J, Neyens JC, van Rossum E, et al. Predicting ADL disability in community-dwelling elderly people using physical frailty indicators: a systematic review. BMC Geriatr 2011;11:1.

42. Schmid A, Duncan PW, Studenski S, et al. Improvements in speed-based gait classifications are meaningful. Stroke 2007;38:2096-100.

43. Brehm M, Bus SA, Harlaar J, et al. A candidate core set of outcome measures based on the international classification of functioning, disability and health for clinical studies on lower limb orthoses. Prosthet Orthot Int 2011;35:269-77. 